\title{
Cuerpo, género e identidades. (Re)visiones a partir de la Psicología institucional
}

\section{Body, gender and identities. (Re)visions from institutional psychology}

\author{
María Eugenia Velázquez \\ Universidad Nacional de La Plata, Buenos Aires, \\ Argentina
}

\begin{abstract}
Resumen
En este artículo se revisan las significaciones imaginarias sociales centrales de género, cuerpo e identidad a partir de la metodología característica de la psicología institucional. Esta es considerada un campo profesional y una perspectiva de construcción de conocimiento aplicable a cualquier ámbito, lo que la convierte en especialmente apropiada para visibilizar la forma en que funciona la construcción colectiva de las significaciones imaginarias sociales, que influyen sobre los modos de hacer y entender la realidad. Se realiza un breve recorrido histórico sobre los conceptos tradicionales de género, cuerpo e identidad y también se intenta dar cuenta de algunas posibles derivas, que se plantean en la actualidad, a partir de su cuestionamiento y deconstrucción. Se trata de visibilizar cómo funcionan estos sentidos y las transformaciones que han sufrido a lo largo del tiempo. Al ser significaciones de primer orden, los sentidos asociados al género, cuerpo e identidad condicionan los modos de hacer en las prácticas profesionales y en la vida corriente. Si estos se sostienen sobre categorías sociales heteronormativas, binarias y cis-sexuales, se entiende cómo han influido en los modos particulares de concebir las conceptualizaciones básicas de la Psicología al patologizar la diferencia y vulnerar los derechos humanos de las disidencias sexuales. Es urgente la revisión de la disciplina desde una perspectiva de género. Este trabajo da el puntapié inicial e invita a que se continúe con otras conceptualizaciones básicas.
\end{abstract}

Palabras clave: género, cuerpo, identidad, significaciones imaginarias sociales, Psicología institucional, Trans*

María Eugenia Velázquez; Universidad Nacional de La Plata, Argentina.

La correspondencia en relación con este artículo se dirige a María Eugenia Velázquez, Universidad Nacional de La Plata, Argentina. Correo electrónico: mevtat@gmail.com 


\title{
M. VeLÁZquez
}

\begin{abstract}
In this article, the central imaginary social meanings of gender, body, and identity are reviewed based on the characteristic methodology of institutional psychology. This is considered a professional field but also a knowledge-building perspective applicable to any milieu, appropriate for visualizing the way in which the social construction of imaginary social significations works and influences ways of being and understanding reality. The traditional concepts of gender, body, and identity are reviewed in a short historical overview, as well as current drifts from the questioning and deconstruction thereof. The aim is to visualize how these senses work and the transformations they have undergone over time. Being central significations, the ways of understanding gender, body, and identity determine behavior in professional practices and in everyday life. As sustained on heteronormative, binary, and cis-sexual social categories, it is understood how they have influenced the particular ways of conceiving the basic conceptualizations of psychology, pathologizing difference and violating the human rights of sexual diversity. There is an urgent need to review the discipline from a gender perspective. This monograph provides the starting point and invites a furtherance with other basic conceptualizations.
\end{abstract}

Keywords: Gender, Body, Identity, Imaginary Social Significations, Institutional Psychology, Trans*

El colectivo travesti-trans* en Latinoamérica, y en el mundo en general, vive situaciones cotidianas de vulneración a sus derechos. Entre ellas se encuentra la dificultad para el acceso a la educación y al sistema de salud, la falta de acceso la vivienda, el no poder ejercer otro trabajo más que el de la prostitución y la discriminación y el maltrato al que se ve sometido en los espacios públicos. Esta exclusión social, producto de una violencia económico-política, sistemática y estructural ejercida sobre el colectivo ha redundado en una disminución notable del promedio de vida que ronda los 35 años de edad para las mujeres trans en el continente, según la Comisión Interamericana de derechos Humanos (CIDH) (Organización de Estados Americanos [OEA], 2018) en tanto que el promedio de vida del resto de la población supera los 80 años.

Se entiende por travestis y trans* a aquellas personas cuya percepción del propio género no se corresponde con:

el sexo asignado al momento del nacimiento[...] (Su expresión de género) puede involucrar la modificación de la apariencia o la función corporal a través de medios farmacológicos, quirúrgicos o de otra índole, incluyendo la vestimenta, el modo de habar y los modales (Ministerio de Justicia y Derechos Humanos de la Nación. Secretaría de Derechos Humanos, 2012, Ley 26.743, articulo 2).

La palabra trans* es acompañada por un asterisco para indicar que el término engloba una pluralidad que no se reduce a la simple abreviación de "transexual" o "transgénero". Manifiesta el carácter situado (Harawey, 1995) y plural de las identidades que comprende. Se incluyen características del momento sociohistórico particular y los contextos locales específicos.

Históricamente, la Psicología ha colaborado en la vulneración del colectivo mediante la patologización de las identidades disidentes. Por eso, desde un marco ético-político se cree que es necesaria la revisión de sus modelos conceptuales en general - y de algunos conceptos específicos en particular- no solo para adecuarlos a los marcos normativos actuales, sino también para evitar la patologización de la 
diferencia que redunda en la vulneración de los derechos humanos de las minorías. Asimismo, dicha revisión debe realizarse a partir de la perspectiva de la psicología institucional, porque no es solo un campo de la Psicología, sino también una perspectiva de construcción de conocimiento.

La Psicología institucional nació en la década de 1960, en Argentina, de la mano de José Bleger (1999) y Fernando Ulloa (1995). Si bien utiliza conceptos provenientes de distintas disciplinas para intentar romper con la antinomia individuo-sociedad, se parte del supuesto ontológico de que la realidad es una construcción sociohistórica constituida por significaciones imaginarias sociales (Castoriadis 2013) en la que los individuos son tanto producto como productores. La institución total de la sociedad es entendida como una trama de instituciones parciales - es decir el lenguaje, las normas, los modos de entender y hacer sobre el mundo, los mitos, las creencias, los valores, los ritos, etc.- que vinculan a los sujetos con la sociedad. Están constituidas por múltiples significaciones imaginarias sociales que funcionan como un magma, al otorgar unidad y, a la vez, dirigir y orientar la vida social. Son significaciones que en tanto que son arbitrarias, dadas por creación, se les identifica como imaginarias, porque no corresponden a elementos "racionales" o "reales". A su vez, es necesario que sean compartidas por un colectivo. El movimiento magmático refiere a una continua transformación, que desplaza permanentemente sus significaciones a otras significaciones; sin embargo, mantiene una coherencia interna que le da identidad propia, un eidos, que facilita que se le vea como una y la misma sociedad.

Estas significaciones imaginarias sociales son performativas en tanto tienen efectos materiales sobre los modos en que se percibe y se actúa sobre el mundo como la psique y el cuerpo de los individuos. Kaës (1989) toma el modelo de la pulsión freudiana para dar cuenta de la cualidad de Janos bifrontes de las intuiciones; es decir, son formaciones intermediaras apoyadas sobre lo subjetivo y lo social, que pertenecen a ambos espacios al mismo tiempo.

La institución no es solamente una formación social y cultural compleja. Al cumplir sus funciones correspondientes, realiza funciones psíquicas múltiples para los sujetos singulares, en su estructura, su dinámica y su economía personal. Moviliza cargas y representaciones que contribuyen a la regulación endopsíquica y aseguran la base de la identificación del sujeto al conjunto social; constituye [...] el trasfondo de la vida psíquica en el que pueden ser depositadas y contenidas algunas partes de la psique que escapan a la realidad psíquica (Kaës, 1989, p. 25; cursivas en el original).

En ese sentido, puede visualizarse cómo, tanto la sociedad como las subjetividades, no son dadas de una vez y para siempre, sino que están en permanente transformación.

La metodología de trabajo de la Psicología institucional consiste en ubicar las significaciones imaginarias sociales (Castoriadis 2013). Los sentidos que se ponen en juego en una práctica (Schejter, 2018) posibilitan identificar cuáles son los efectos que estos sentidos tienen sobre la realidad concreta de las personas y su modo de entender y actuar sobre el mundo. "La entendemos (a la psicología institucional) como un enfoque epistemológico de lo psicológico que pone en relación las instituciones que atraviesan tanto a los sujetos como agrupamientos, con el modo en que estos entienden y sostienen sus prácticas." (Schejter, 2018, p. 13). La Psicología institucional se ubica como disciplina articuladora entre el común colectivo y lo psíquico individual. Hace lecturas propias que permiten romper con la heredada y falaz antinomia individuo-sociedad, dando cuenta - aunque siempre inacabadamente- de 
los vínculos complejos entre estas dos instancias, prontas a descubrir que son impensables la una sin la otra. La ambivalencia del concepto de institución (Malfé, 1982), el cual no coincide exactamente con el concepto de organización, da cuenta de que el objeto de la psicología institucional trasvasa los límites del lugar y la situación específica que se estudia para realizar un análisis complejo, multidimensional, crítico y localizado (Harawey, 1995). De allí, surge la importancia de analizar los conceptos de género, cuerpo e identidad a partir de la Psicología institucional; es decir, al utilizar la metodología propia de este enfoque en función de deconstruir, (re)conceptualizar y (re)visar cómo se ha llegado hasta aquí.

Antes de entrar de lleno en el análisis, es importante señalar que tradicionalmente las sociedades se han manejado con significaciones imaginarias sociales que establecen como algo "natural" el binarismo sexual — solo dos sexo/géneros, complementarios y excluyentes—, la heteronormatividad - y la cis-sexualidad — se da por supuesto que la identidad de género coincide necesariamente con el sexo biopolíticamente asignado al nacer-. Sin embargo, la lucha política de las disidencias sexuales ha logrado ir instalando otra lógica. Generalmente, las personas se manejan con significaciones imaginarias sociales basadas en el binarismo sexual y entienden el sistema sexo-genérico como una combinación de los elementos de la figura 1 .

Figura 1.

Sistema sexual binario.

\begin{tabular}{|c|c|c|c|}
\hline Sexo biológico & $\begin{array}{c}\text { Identidad sexual/de } \\
\text { género }\end{array}$ & Orientación sexual & Expresión de género \\
\hline $\begin{array}{c}\text { Genitales: vulva/pene } \\
\text { Hormonas: } \\
\text { testosterona/estrógeno } \\
\text { Cromosomas: }\end{array}$ & $\begin{array}{c}\text { Cis-género } \\
\text { Transexual }\end{array}$ & $\begin{array}{c}\text { Heterosexual } \\
\text { Homosexual/esbiana } \\
\text { Bisexual }\end{array}$ & $\begin{array}{c}\text { Masculino } \\
\text { Femenino }\end{array}$ \\
\hline
\end{tabular}

Nota. La figura sintetiza en conceptualizaciones trabajadas en el video “¿Qué es la diversidad sexual? Explicación fácil” (Carki productions, 2018).

Es decir, se supone que hay elementos biológicos — genitales, hormonas y cromosomas- que condicionan la división en dos sexos claros y distintos, que, a su vez, coinciden con la expresión de género masculina y femenina — cis-sexualidad-. Las posibilidades de disidencia de estos aspectos serían que una persona no se identifique con el género asignado al nacer y "migre" a una expresión de género coincidente con la del sexo opuesto - transexualidad- O bien, que su orientación sexual se vea "invertida" y se sienta atraída por personas de su mismo sexo-género — lesbianismo y homosexualidad-o bien por ambos - bisexualidad-. Desde esta perspectiva y a partir de la heteronormatividad obligatoria, el género-sexo y la orientación sexual son coincidentes. Se espera que una mujer — cis o trans- se vea "naturalmente" atraída por varones y viceversa. 
Las luchas del colectivo LGBTIQA+ plantean, en cambio, que el sistema sexual es diverso y amplio. Su espectro comprende las múltiples combinaciones que permiten los elementos que aparecen en la figura 2.

Figura 2

Diversidad sexual.

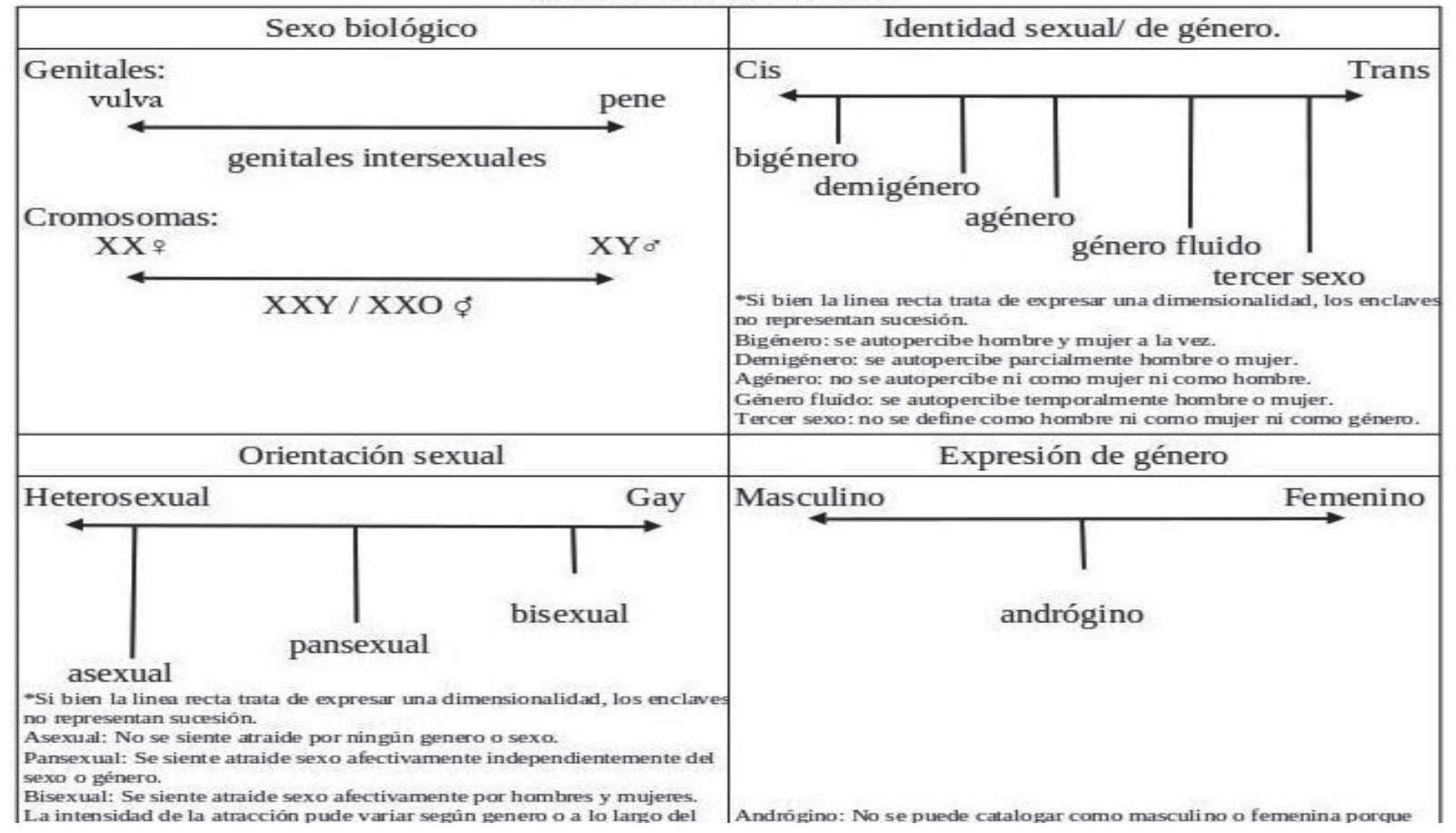

Nota. La figura sintetiza en conceptualizaciones trabajadas en el video “¿Qué es la diversidad sexual? Explicación fácil” (Carki productions, 2018).

Es necesario señalar que este último cuadro se modifica permanentemente y que el modo gráfico no permite dar cuenta del amplio abanico que conforma cada subjetividad en el espectro de la sexualidad humana. Desde esta otra perspectiva, se intenta visibilizar que los componentes biológicos no demarcan una división entre los sexos tan clara. Por el contrario, existen personas intersexuales que manifiestan características genitales ambiguas. Hay combinaciones cromosomáticas diferentes a las tradicionales, como podría ser el XXO o el XXY, y que, en lo que respecta a las hormonas, todas las personas producen tanto testosterona como estrógeno en distintas proporciones. La identidad de género incluye otros modos de identificarse más allá de la cis-sexualidad o la transexualidad; por ejemplo, el bi-género que se percibe al mismo tiempo como varón y mujer o el a-género que no se percibe ni como hombre ni como mujer, 
entre otros múltiples posibles. La orientación incluye — entre otras - también la asexualidad, es decir, aquellas personas que no sienten atracción sexual por otras o la pansexualidad para quienes la atracción está desvinculada del género de la persona que se desea. Finalmente, la expresión de género no es solo masculina o femenina. Hay toda una dimensionalidad que puede ponerse en juego en las distintas combinaciones de elementos tradicionalmente asignados a alguno de los géneros, como también la androginia que implica una presentación social que es genéricamente ambigua. Este segundo cuadro simplemente trata de ilustrar el sistema de la diversidad sexual que incluye también a los modos más tradicionales de entender la sexualidad y el género. A partir de la visibilización de las diferentes posibilidades, se plantea una resignificación de las estructuras sociales para crear una lógica que rompa con "lo uno" y "lo otro" (Beauvoir, 2017), dando lugar a la diversidad. En la convivencia de ambos cuadros, las significaciones imaginarias sociales (Castoriadis, 2013) pueden ser entendidas a partir de la metáfora del magma, porque constituyen un conjunto heterogéneo de sentidos que coexisten en una misma sociedad. Incluso, llega a contradecirse entre sí sin constituir mayores problemas para la cohesión colectiva.

Si la realidad es una construcción social orientada con base en significaciones imaginarias sociales heteronormativas, binarias y cis-sexuales, es muy probable que estos supuestos se hayan dado por sentado influyendo, silenciosa pero eficazmente, en los modos tradicionales de concebir el género, el cuerpo y la identidad. Sus consecuencias son concretas y se padecen en los cuerpos de las disidencias que han sido históricamente patologizadas, excluidas y discriminadas. Tomando esto en consideración, se realizará un recorrido bibliográfico por los modos pretéritos y contemporáneos de entender los conceptos de género, cuerpo e identidad, utilizando la metodología propia de la Psicología institucional para poder identificar cómo se han ido concibiendo y transformando a lo largo del tiempo. Es importante señalar que no se trata de una revisión sistemática de la bibliografía referida a estos temas, sino de un estudio que toma algunos escritos como material de análisis tratando de identificar las significaciones imaginarias sociales (Castoriadis, 2013), los sentidos que sostienen un modo específico de entender el género, el cuerpo y las identidades en cada uno de esos textos que configuran mojones a lo largo del tiempo, y que mediatizan un modo específico de entender la realidad y actuar sobre ella, incluyendo los efectos concretos que ese modo particular de entender la realidad tiene sobre la vida, lo cuerpos y la psique de las personas. A su vez, es preciso recalcar que fueron las luchas LGTBIQA+ las que señalaron la vulneración de derechos humanos y la función opresiva que ha tenido la Psicología y otras ciencias a lo largo de los años sobre esta comunidad. No fue una revisión crítica de la propia disciplina la que introdujo estos cambios y, por ende, en muchas oportunidades en Psicología se siguen manteniendo posturas ostracistas que resisten al cambio y vulneran derechos para las diversidades. Esta coyuntura actual en la que conviven perspectivas no siempre reconciliables es suficiente para que esta revisión conceptual resulte necesaria y urgente.

\section{¿Qué es el cuerpo?}

Ya se señaló más arriba que las significaciones imaginarias sociales (Castoriadis, 2013) generalmente circulan en la sociedad y se corresponden al binarismo sexo-genérico, que suele entenderse como "natural" y que además se tienden a superponer los conceptos de orientación sexual, sexo y género. Sin embargo, estas concepciones aparecieron solo después de siglo XIII. Antes de esa época, el modo de entender las diferencias sexuales era metafísico (Schiebinger, 2004). Entonces, se creía que hombres y mujeres contaban con los mismos órganos sexuales. Los preceptos médicos aristotélico-galénicos 
indicaban que las mujeres, por falta de calor, no habían logrado expulsar los genitales al exterior. Por esta razón, tenían el pene alojado en el bajo vientre y se creía que los ovarios eran testículos internos. De hecho, se les denominaba de la misma manera: "piedras". Así, las mujeres eran consideradas un ser inferior, aunque no se descartaba -incluso se documentaban casos-, que se pudieran expulsar los órganos sexuales hacia el exterior y se convirtieran inmediatamente en hombres. Galeno (2003, como se citó en Schiebinger, 2004) no consideraba que existiesen diferencias genitales:

Todas las partes que tienen los hombres las tienen también las mujeres [...] la diferencia entre ellos radica en una sola cosa [...] que en la mujer las partes están dentro del cuerpo, mientras que en el hombre están fuera (p. 239).

Con el Iluminismo recién aparecen las significaciones imaginarias sociales (Castoriadis, 2013) de la noción de sexo tal como la entendemos hasta nuestros días. "A fines del siglo XVII y en el XVIII, la ciencia otorgó una sustancia material, aceptables en términos de la nueva epistemología, a las categorías de 'hombre' y 'mujer', consideradas como sexos biológicos opuestos e inconmensurables" (Laqueur, 1994, p. 266).

Los órganos comienzan a diferenciarse lingüística y anatómicamente y se empieza a dar nombre específico a aquellas partes que hasta el momento no lo tenían; por ejemplo, la vagina. De un extremo se pasa al otro, de la indiferenciación absoluta a una distinción anatómica específica y universalizable para las categorías opuestas y complementarias "hombre" y "mujer". A las personas intersexuales se les considera "desviaciones" y se plantea la necesidad de "normalizar" sus genitales a partir de las cirugías de resignación de sexo durante los primeros meses de vida. Se entiende como fundamental que se puedan identificar en forma precisa si los genitales de una persona son masculinos o femeninos, porque cualquier ambigüedad es absolutamente disruptiva (Di Segni, 2013). Así, podría decirse que se entiende por organismo las conceptualizaciones racionalizadas que han desarrollado las ciencias biológicas para tratar de explicar cómo funciona un cuerpo (Leibson, 2018).

En “¿Cómo se forma un cuerpo? Hacia una problematización sociocultural de la noción de cuerpo desde la tensión naturaleza cultura", Gambarotta y Mora (2018) plantean que la noción de cuerpo en las ciencias sociales tiende a ser un presupuesto en los estudios sobre esta temática. Si bien la mayoría de estas investigaciones hablan de la construcción social del cuerpo, lo que según estos autores se desliza en los trabajos científicos es que llega un momento en que el cuerpo se "naturaliza". Al estudiar la danza, las emociones o lo no-conceptual como parte de los estudios del cuerpo, no se problematiza esta relación, sino que se establece como dada.

Estamos, en última instancia, ante una substancialización del cuerpo o, mejor aún, ante lo que cabe entender como su naturalización. Es decir, el proceso por el cual se establece al cuerpo como una suerte de 'segunda naturaleza', que escapa a las determinaciones culturales y prácticas, tanto como la 'primera' (Gambarotta \& Mora, 2018, p. 15).

Se puede observar en esta cita cómo los sentidos que se ponen en jugo en las significaciones imaginarias sociales (Castoriadis, 2013) referentes al cuerpo lo toman como algo sustancialmente natural. Es decir, cómo la tradición de categorías binarias, opuestas y excluyentes que divide y opone el cuerpo - lo 
"natural" - y la cultura - lo social — se filtra subrepticiamente en los supuestos de los investigadores y termina teniendo efectos no conscientes en el modo particular de entender el cuerpo desde las ciencias sociales.

Freud (1893/1976) entiende al cuerpo no como una máquina biológica que responde a las leyes de la naturaleza, sino como una dualidad, una metáfora. Lo primero que nota en las histéricas que estudia es que sus parálisis no responden a la "anatomía cerebral verdadera" (p. 205), sino "que es la concepción trivial, popular, de los órganos y del cuerpo en general la que está en juego en las parálisis histéricas" (p. 207). A partir de estos síntomas y de la ausencia de lesión física, Freud pergeña la idea de que hay un otro cuerpo, que desconoce al biológico y que está constituido por representaciones vinculadas entre sí. Pero, no se queda allí. Plantea, a su vez, que los lazos establecidos entre las representaciones funcionan en relación con un "valor afectivo" (p. 208). Es notable que no abandona la idea de lesión, sino que propone que lo que se encuentra dañado es relacionado con la representación.

Si el cuerpo, o al menos un cuerpo, es un conjunto de representaciones, puede ser pensado como la proyección de una superficie. Este cuerpo hecho de representaciones está configurado de forma distinta para cada sujeto, se construye en función de los términos vulgares de los órganos y desconoce la anatomía científica. De la misma forma, implica su imagen como proyección de una superficie, la cual se constituye también como una superficie. Y, a su vez, esta imagen proyectada brinda una falsa sensación de unidad. Lacan (2003) llama a este momento estadio del espejo. Se refiere a la primera ocasión en el que el infans, que aún no tiene un control psico-motriz efectivo sobre sus miembros, ve su reflejo en el espejo y comprende que esa imagen que percibe es él mismo, que tiene una entidad y puede diferenciarse tanto de los objetos que lo rodean, así como del Otro que lo sostiene. La identificación con la imagen reflejada en el espejo produce la anticipación del control que podrá ejercer sobre sus miembros y una percepción gestáltica de unificación a la que se aliena. En ese momento, se produce en el niño una expresión de júbilo que se dirige hacia el Otro que lo sostiene, quien, con un gesto afirmativo, ratifica las percepciones del niño. Queda establecida así "la matriz simbólica en la que el yo [je] se precipita en una forma primordial, antes de objetivarse en la dialéctica de la identificación con el otro y antes de que el lenguaje le restituya en lo universal su función de sujeto" (Leibson, 2018. p. 87). Es decir que, en este momento, las sensaciones corporales que hasta entonces se le presentaban como fragmentarias —real—, comienzan a ordenarse en función de la sensación de unidad que prefigura el yo [je] -imaginario- y de la anticipación del control que prefigura el sí mismo [moi] — simbólico.

Se puede identificar que aquí se advierte algo de lo corporal que quedaría por fuera de lo simbolizable, en términos de Lacan, lo real. Pero, se diferencia de lo que Gambarotta y Mora (2018) denominan como "naturalización" del cuerpo. Sin embargo, mantiene la característica de concebir al cuerpo a partir de significaciones imaginarias sociales que implican categorías excluyentes entre sí. Para evitar la despolitización de la que nos advierten estos autores, se puede hablar del cuerpo en lo simbólico desde otra perspectiva. Específicamente, desde la concepción de habitus (Bourdieu \& Wacquant, 2014). La lógica de los campos es un modo particular de leer la dinámica social en un momento determinado. Se trata de una metodología praxeológica que intenta romper, por un lado, con un punto de vista fisicalista, desde el cual la sociedad es concebida como una estructura objetiva independiente de los actores que participan en ella, y en la que estos pueden ser pensados como soportes pasivos que "actúan" las fuerzas que se ejercen sobre ellos. Por el otro, con un punto de vista fenomenológico, en el que "la sociedad aparece 
como el producto emergente de las decisiones, acciones y cogniciones de individuos cocientes y alerta, a quienes el mundo se les presenta como inmediatamente familiar y significativo" (p. 33). Para lograrlo, Bourdieu (Bourdieu \& Wacquant, 2014) plantea un sistema bidimensional en el que hay una correspondencia entre las estructuras sociales y los principios de interpretación y acción que los agentes aplican sobre el mundo. La estructura del campo se refleja o duplica en habitus de los agentes, disposiciones mentales y corporales que orientan la forma de entender y actuar en un campo determinado, con sus dinámicas particulares de conflicto y sus luchas simbólicas por el sentido. En este plano se (re) construyen y se instituyen las clasificaciones del campo. Así, quedan "enclasadas" simbólicamente.

En "la dominación masculina" (Bourdieu, 2000) se plantea que el habitus de género trasciende los campos.

La división entre los sexos parece estar «en el orden de las cosas», como se dice a veces para referirse a lo que es normal y natural, hasta el punto de ser inevitable: se presenta a un tiempo, en su estado objetivo, tanto en las cosas (en la casa por ejemplo, con todas sus partes «sexuadas»), como en el mundo social y, en estado incorporado, en los cuerpos y en los habitus de sus agentes, que funcionan como sistemas de esquemas de percepciones, tanto de pensamiento como de acción.[...] La concordancia entre las estructuras objetivas y las estructuras cognitivas, entre la conformación del ser y las formas del conocer, entre el curso del mundo y las expectativas que provoca, permite la relación con el mundo que Husserl describía con el nombre de «actitud natural» o de «experiencia dóxica», pero olvidando las condiciones sociales de posibilidad (p. 10; cursivas y comillas en el original).

Así, se puede observar el proceso social de masculinización o feminización de los cuerpos, los cuales adquieren características — modos de vestirse, andar, emociones que se pueden mostrar o no, etc.- que tienen sentido dentro de las clasificaciones de lo femenino y lo masculino, las cuales, a su vez, están jerarquizadas. Se da, entonces, una somatización de la dominación propia de las relaciones sociales ente hombres y mujeres. Este modo particular de entender las relaciones entre el cuerpo y el género, se corre un poco de las significaciones imaginarias sociales (Castoriadis, 2013) establecidas con anterioridad. Plantea una relación complejizada entre lo social y lo corporal, que ya no puede tomarse como aspectos separados y excluyentes, sino que la relación es íntima y de difícil diferenciación. Comienza a verse algo de los cambios en estas significaciones, que tiene efectos transformadores en los modos de entender el cuerpo.

Esta construcción social y subjetiva de los cuerpos generizados se ha realizado en parte mediante la indumentaria. Zambrini (2010) en "Modos de vestir e identidades de género: reflexiones sobre las marcas culturales en el cuerpo" realiza una construcción sociohistórica de cómo la moda ha ayudado a naturalizar las identidades de género binarias y la matriz heteronormativa al considerar los cuerpos socialmente legibles a partir de su apariencia. Allí, señala que en las etapas anteriores a la emergencia de la burguesía, la indumentaria era utilizada para marcar una diferencia de status. Mediante las Leyes Suntuarias, se impedía la utilización de ciertas telas, colores y prendas a personas que no formaran parte del clero, la realeza o la corte. Recién a partir de la mitad del siglo XIX, la moda comienza a reforzar la división entre los modos e imaginarios de lo masculino y lo femenino. A este momento sociohistórico, 
se le denominó “La Gran Renuncia”, puesto que el vestuario masculino perdió su función ornamental y quedó instalada la figura del $d a n d y$ como modelo privilegiado. La uniformidad y la sobriedad fueron favorecidas en la indumentaria masculina, mientras que en la femenina se intentaban destacar las formas y la sensualidad consideradas atributos de las mujeres.

La clasificación estética del mundo supone una permanente tensión y negociación respecto a cuáles identidades son susceptibles de ser visibilizadas legítimamente, y cuáles se transforman en diferencias u otredades. Porque las prácticas del vestir, en tanto discurso estético, hacen referencia a la comunicación y clasificación social de los cuerpos generizados, haciéndose inteligibles en términos identitarios para la mirada de la sociedad (Zambrini, 2010, p. 144).

\section{¿Qué es el género?}

Como puede observarse en el apartado anterior, las significaciones imaginarias sociales (Castoriadis, 2013) hasta hace relativamente poco igualaban el sexo - que desde la perspectiva de categorías excluyentes correspondería al cuerpo- con el género - que desde esa misma perspectiva correspondería a lo social o cultural. En "Sexualidades: tensiones entre la psiquiatría y los colectivos militantes" (Di Segni, 2013), se rastrean los diferentes modos sociohistóricos de entender la sexualidad. La primera persona que conceptualiza el "género" es John Money en 1972, quien lo separaba del concepto de "sexo". Este psicólogo realizaba estudios con niños intersexuales y planteaba que el género era el modo en que se expresaban comportamientos que servían para asignar socialmente un estatus de varón o de mujer a cada persona. Proponía que, luego de realizar una cirugía genital que desambiguara el sexo del niño tratado y hasta los 18 meses de edad, era posible "inscribir" el género deseado sin mayores dificultades. Money no cuestionaba la noción binaria de los sexos. Por el contrario, trataba de reforzarla. Sin embargo, el "caso Joan/John" fue emblemático en tanto que demostró falsas las teorías de Money. David, uno de los gemelos Reimer, sufrió una amputación accidental del pene mientras le realizaban la circuncisión y fue reasignado mujer quirúrgicamente. El joven fue criado bajo estrictas indicaciones del psicólogo, se retiraron todos los indicios de su antiguo género y en la adolescencia se le suministraron hormonas correspondientes al sexo reasignado. Sin embargo, su autopercepción siempre fue la de un varón.

Lo que Money dejaba en claro al justificar la cirugía temprana para enmendar a la naturaleza era que esta producía variedad (no binarismo) sobre la cual la cultura/medicina imponía su marca: si la naturaleza cumplía con las normas impuestas por la Cultura y custodiadas por la Medicina, se la dejaba en paz; si esto no ocurría, se la corregía (Di Segni, 2013, p.240).

En el ámbito académico, hasta el presente, ha habido un gran desarrollo de los llamados estudios de género, los cuales intentan dar cuenta de las diferencias estructurales entre varones y mujeres en función de generar una mayor igualdad entre estos, los cuales, progresivamente, han ido incluyendo a las disidencias sexuales. Desde este ámbito, surgen los estudios trans*, un área del conocimiento específicamente orientada al trabajo con personas travesti-trans*.

En un primer momento, como se vio con Di Segni, la transexualidad era considerada una patología tanto por el ámbito médico como el de la salud mental. De hecho, solo a partir de 2013 el "Trastorno de la identidad de género" desapareció del DSM-5 (American Psychiatric Association, 2014) y es remplazado por el "trastorno de disforia de género". En "Del transexualismo a la disforia de género en el DSM. 
Cambios terminológicos, misma esencia patologizante", Mas-Grau (2017) propone que los cambios en este reconocido manual se plantean a partir de tensiones entre los redactores y distintos sectores de lucha por los derechos trans*, a partir de las cuales, si bien se modifica la nomenclatura, se siguen manteniendo connotaciones mórbidas en la categoría diagnóstica. En esta línea, también se inscribe el número monográfico de "Quaderns de Psicologia" titulado "Psicología e identidades transgénero en el contexto Iberoamericano" (Martínez-Guzmán \& Prado, 2015) en el que se publican nueve trabajos monográficos que plantean la relación entre las formas en las que la psicología ha impactado y definido las trayectorias vitales y las identidades transgénero, y las aproximaciones empíricas y teóricas que intentan comprender la experiencia y la perspectiva subjetiva trans* desde la voz de sus protagonistas. Como se señaló antes, aquí, pueden observarse las resistencias al cambio propias del ámbito de la Psicología, especialmente al considerar que estos no se producen por una revisión crítica de la propia disciplina, sino que vienen impuestos por transformaciones en las significaciones imaginarias sociales (Castoriadis, 2013) propiciadas por las luchas políticas desde una perspectiva de derechos de los movimientos LGTBIQA+.

La mera existencia de la población trans* cuestiona el sistema binario sexo-género y pone en tela de juicio los modos tradicionales de entender la identidad como algo intrínseco y estable. Butler (2002, 2007) piensa críticamente las relaciones entre sexualidad y cuerpo e intenta dar cuenta de que los géneros son una construcción social que se performativiza diariamente en las acciones y representaciones que se llevan a cabo al reproducir un discurso de poder/saber. En "El desafío trans. Consideraciones para un abordaje situado de las identidades de sexo/género" (Martínez-Guzmán \& Montenegro, 2011) se abordan conceptualizaciones críticas sobre la identidad, se realiza un mapeo sobre la diferencia y multiplicidad de posiciones políticas e identitarias al interior de la cuestión trans* y se sitúa a figura de "trans-conocimiento" en el intento de plantear una perspectiva situada (Harawey, 1995) para escapar a las teorizaciones homogeneizantes y permitir un abordaje de la coexistencia transformadora de la multiplicidad.

En la Argentina, Martínez (2015) presentó su tesis de doctorado en la Facultad de Psicología de la Universidad Nacional de La Plata titulada "Identidad y cuerpo. Auto-percepciones de sujetos no conformes al género" en la que "pretende indagar los modos en que sujetos no conformes al género se autodenominan, cómo recrean desde un sentido singular tales categorías y qué lugar ocupa el cuerpo en estas auto-percepciones en torno al género" (Martínez, 2015. p. 10). Allí, plantea que las personas entrevistadas continúan significando su propia experiencia bajo los términos normativos de la lógica binaria y sustancial del cuerpo, propia del modo contemporáneo en que actualmente se ordenan los géneros. Aunque las localizaciones de sujeto no conformes al género no escapan a la égida de los estereotipos, sí encuentra que el género sentido o vivido por los sujetos - aquel que sienten ser-genera una paradoja respecto al género hacia el que transitan. Esta brecha, cuya sutura permitiría el logro de la autenticidad de género, parece imposible de saldar debido a que, de modo subterráneo, los sujetos parecen no poder desembarazarse de concepciones naturalistas respecto del propio cuerpo, que se experimenta como un continuo retorno de signos del sexo de partida que es preciso corregir mediante diversas estrategias. Estas investigaciones desarrolladas desde la metodología de la teoría fundamentada (Charmaz, 2013) dan cuenta de cómo las significaciones imaginarias sociales (Castoriadis, 2013) referentes al cuerpo y género condicionan los modos de entender la realidad para la población general y para el colectivos trans* en particular y cómo estas repercuten en como las personas cis entienden e interactúan con las 
personas trans*al discriminarlas y excluirlas. También, cómo las personas trans* entienden su propio cuerpo y los efectos que esto tiene en los procesos psíquicos vinculados a la transición y la autopercepción identitaria.

La ausencia de estadísticas e indicadores representativos de la situación de la población trans* en el continente americano supone una gran dificultad para saber cuál es la realidad de esta población. Los Estados, en términos generales, no se preocupan por esta condición de vacancia, dando cuenta del poco interés que les produce las circunstancias en las que se encuentran las personas trans*. Algunas organizaciones nacionales e internacionales no gubernamentales han desarrollado observatorios y estadísticas que intentan reflejar la situación del colectivo, algo que logran muy parcialmente, porque dependen de financiación externa y carecen de la llegada territorial que puede desplegar un estado. Sin embargo el informe "¡Paren de matarnos!” (Red Latinoamericana y del Caribe de Personas Trans [RedLacTrans], 2020) es una esquematización sobresaliente de la vulneración de derechos humanos de la población trans* en América Latina y el Caribe desarrollado a partir de los datos recabados por las Organizaciones de Base Nacional (OBN) lideradas por personas trans*, emplazadas en 23 países de la región y sistematizado por el Centro de Documentación y Situación Trans en Latinoamérica y el Caribe (CeDoSTALC). "El CeDoSTALC se constituye como un sistema comunitario para recopilar información, monitorear y brindar respuesta a las barreras que impiden el acceso a los derechos humanos de la población trans* en la región. Dicho sistema se ancla en una plataforma virtual de recolección de casos que posibilita la circulación y centralización de la información de las OTBN (Organización Trans de Base Nacional) con la Secretaría Regional de la RedLacTrans" (RedLacTrans, 2020, p.3).

En este informe se indica que en el periodo de 2019 y 2020 en lo que se refiere al derecho a la vida la población trans* de América Latina y el Caribe tiene una de las tasas más altas de muertes por crímenes de odio sin consecuencias judiciales. De estas, el porcentaje más alto (31.5\%) se refiere a muertes causadas por el abandono estatal al que se encuentra sometida esta identidad. En este sentido, es importante entender que las muertes por enfermedades evitables, por falta de acceso a condiciones elementales de vida, por situaciones de extrema vulnerabilidad, entre otras, se considera identicidio (Wayar, 2018) o travesticidio social, es decir "el mecanismo llevado adelante por el Estado y la sociedad a través de la discriminación, la violencia, la segregación, la represión y la violación sistemática de los Derechos Humanos de las personas travestis" (Ministerio Público de la Defensa \& Bachillerato Mocha Celis. 2017, p.139). Respecto al tipo de vulneración de derechos humanos, el informe "¡Paren de matarnos!" (RedLacTrans, 2020) indica que el 42\% refiere a formas de violencia extrema: golpizas, violaciones y asesinato. Mientras que el $25.3 \%$ se registra bajo el ítem "otro tipo", en el que se incluye principalmente la ausencia del Estado como origen directo de la violación de los derechos humanos. El principal lugar en el que se ejercen este tipo de violencias sobre la población trans* de Latinoamerica y el Caribe es la vía pública; es decir, a pesar de ser una circunstancia que podría inhibir a la persona agresora por la gran exposición que implica, esto no resulta un impedimento. Es posible que se deba a que el $40 \%$ de las agresiones son cometidas por personas que pertenecen a entidades que tienen poder de fuego directo sobre las víctimas; es decir, grupos criminales, pero también fuerzas de seguridad, militares y seguridad privada. En lo que se refiere al acceso a la educación, el informe indica que en más del $60 \%$ de los casos registrados las personas trans* han abandonado sus estudios durante la educación primaria. Esto no es 
sorprendente si se tiene en cuenta la falta de reconocimiento legal de las identidades diversas en muchos de los países que forman parte del estudio - lo que afecta directamente al acceso a la educación-, la gran discriminación y la violencia a la que se ve expuesta esta población en los ámbitos educativos, la falta de apoyo familiar - ya que muchas personas trans* son expulsadas de sus hogares en cuanto comienzan tener una presentación social adecuada a la identidad de género autopercibida - y a la falta de políticas estatales que prevean estrategias específicas que faciliten la retención y continuidad educativa del colectivo. En lo relativo al acceso al trabajo, la gran mayoría de mujeres trans* tienen como forma única de sustento e ingresos económicos el trabajo sexual. Si a esto se le agrega que en la mayor parte de la región el trabajo sexual está criminalizado al producir una persecución y violencia institucional por parte de las fuerzas de seguridad, se infiere una doble estigmatización de estas personas: por ser trans* y prostituta. Como se mencionó antes, en muchos de los países que se incluyen en el estudio aun no está reconocido el derecho a la identidad, lo que provoca prejuicios y discriminación social sobre esta población e impide el acceso a trabajos registrados. Asimismo, si se quiere acceder o permanecer en un empleo formal, la persona suele tener que presentarse socialmente en concordancia con las significaciones imaginarias sociales cisexuales que atraviesan a la población, lo que redunda en una "mutilación" de un aspecto fundamental de su subjetividad. "En Latinoamérica y el Caribe a las personas trans* se les vulnera el derecho al trabajo y a todos sus derechos humanos; esto sucede en un contexto de extrema violencia con niveles que no se registran dentro de otras poblaciones" (RedLacTrans, 2020, p. 32).

Marlene Wayar (2018) gesta la teoría trans trava latinoamericana en la que plantea el fracaso de la pedagogía heterosexual y la perspectiva adultocéntrica. En "Travesti. Una teoría lo suficientemente buena" quedan plasmados sus diálogos con la poeta travesti chilena Claudia Rodríguez, la artista trava, sudaca, Susy Shock y la periodista Claudia Acuña, en los que se repiensa críticamente el sistema hegemónico heteronormativizador que excluye todo lo que se perciba como diferente.

Esta teoría nos urge ante la terrible propuesta hegemónica de ser mutaciones antropológicas rumbo a estar al comando de la tecnología de crianza, alimenticia, educativa, sanitaria, comunicacional y, sobre todo, relacional. [...] Esta es una teoría lo suficientemente buena para comenzar a accionar en vistas a una transformación antropológica que nos devuelva autonomía, que nos devuelva la desconfianza necesaria para una crianza con amor responsable, lejos de toda banalidad. Entender el amor responsable es crucial. (Wayar, 2018, p.17)

Las experiencias y las teorías producidas desde las mismas identidades trans* permiten deconstruir y desnaturalizar algo de las significaciones imaginarias sociales (Castoriadis, 2013), que estructuran los modos instituidos de comprender la realidad y filtran encubiertamente categorías binarias, heteronormativas y cis-sexuales.

\section{¿Qué es la identidad?}

La construcción social de los sentidos, o significaciones imaginarias sociales que, en la modernidad y en la modernidad tardía, se encuentran teñidos por los valores positivistas, racionales y binarios, influyó en que se instalaran algunas prácticas y valores socioculturales —en detrimento de otros- al darse como "naturales". Entre ellos se encuentra la construcción de identidades generizadas (Arfuch, 2002) al apuntalar la lucha que implica la construcción de identidades y otredades. En esta línea, puede 
entenderse el género como una tecnología (Foucault, 1989, 1990, 2003). Es decir, los mecanismos sociales generan la tipificación de un cuerpo "normal" que ejerce una presión social que produce un tipo específico de subjetividad a partir de la regulación y control social de los sujetos.

De Lauretis (1989), retomando las conceptualizaciones de Foucault, plantea que el género no es una esencia de los cuerpos, sino un modo de representación y autorrepresentación producto de tecnologías sociales - como el cine-, los discursos institucionales — como las teorías y epistemologías-, así como prácticas sociales y culturales en sentido amplio, los cuales tienen una serie de efectos sobre los cuerpos, los comportamientos sociales y las relaciones a partir del despliegue de estas tecnologías políticas complejas. Estas tecnologías serían los medios por los que se transmiten y reproducen las significaciones imaginarias sociales tal como las piensa Castoriadis (2013).

En la misma línea, se inscriben las conceptualizaciones de Preciado (2008) sobre códigos semiótico técnicos. Los dispositivos de producción subjetiva que planteara Foucault han pasado de ser externos y rígidos a blandos e internalizados. Los dispositivos ya no controlan los cuerpos desde fuera, sino que son asimilables, productos farmacopornográficos que los invaden a nivel de transformarlo en un tecno-cuerpo al construir literalmente el cuerpo que (no) somos.

Hall (2003) plantea que las identidades no se construyen fuera del discurso sino dentro de él —vale decir de las significaciones imaginarias sociales (Castoriadis, 2013) - y que, por lo tanto, es necesario considerar su producción en el contexto institucional e histórico específico con prácticas y formaciones discursivas específicas mediante estrategias enunciativas específicas. A su vez, surgen en un interjuego específico de poder; por lo tanto, "son más un producto de la marcación de la diferencia y la exclusión que signo de una unidad idéntica y naturalmente constituida: una «identidad» en su significado tradicional" (p. 18; comillas en el original). Este autor plantea que la identidad es un concepto "que funciona «bajo borradura»" (p. 14; comillas en el original). Es decir, que luego de las lecturas posestructuralistas y deconstructivistas, ya no remite al significado original de identidad unificada, originaria e integral, pero tampoco han nacido nuevas conceptualizaciones que puedan remplazarlo como concepto que "ayude a pensar".

La identidad sería entonces no un conjunto de cualidades predeterminadas - raza, color, sexo, clase, cultura, nacionalidad, etc. - sino una construcción nunca acabada, abierta a la temporalidad, la contingencia, una posicionalidad relacional sólo temporariamente fijada en el juego de las diferencias (Arfuch, 2002. p.24).

Butler (2007) plantea que la identidad de género crea la ilusión de una sustancia interior y profundamente inmanejable. Propone, por el contrario, al género como un acto políticamente impuesto con sutileza. La performatividad del género implicaría un ritual, una repetición que tiene efectos mediante la naturalización en el cuerpo. Una "duración temporal sostenida culturalmente" (p. 17). Las personas trans* pondrían en cuestión esta naturalización del género, es decir, las significaciones imaginarias sociales (Castoriadis, 2013) binarias que suelen adjudicarse al género. En tanto, los cuerpos generizados — binariamente - pueden leerse y son construidos socialmente, es factible utilizar distintos tipos de tecnologías que faciliten una lectura adecuada al género autopercibido. Las tecnologías estéticas (CastañónVázquez, 2018) son todas aquellas herramientas relacionadas con la indumentaria, el cuidado capilar y el maquillaje que pueden utilizar las personas trans* para su transición. Asimismo, la tecnología médica 
ha permitido en el contexto occidental contemporáneo la posibilidad de transformar el género atribuido biopolíticamente al nacer a través de cirugías y tratamientos hormonales. Se entiende por transición "el proceso de transformación emprendido por parte de personas trans para lograr la que consideran una correcta atribución de género por sí y por parte de los demás" (Garosi, 2012, p. 141). En este sentido, el género y la transición constituyen un proceso interactivo y social que se desarrolla para tener una atribución del género adecuada para sí y para otros. Si bien esta última autora plantea que la transición se realiza en tres niveles: (a) el cuerpo, (b) el sexo y (c) los roles sociales - generizados-, también indica que la misma puede derivar en (a) la reproducción de los patrones dominantes del género, (b) la innovación, entendida como las transformaciones que no se corresponden con la expectativa social de un "correcto" cuerpo sexuado y (c) la superación del esquema binario del género, al independizar el sexo de la biología y reclamar la multiplicidad de géneros. Es importante señalar que los modos de pensar la identidad más cercanos en el tiempo a la actualidad cuestionan e interpelan las categorías tradicionales de género y cuerpo. Sin embargo, se está muy lejos de que estas sean significaciones o sentidos que circulan en la población general. Los procesos por los cuales las significaciones imaginarias sociales (Castoriadis, 2013) se modifican pueden ser muy largos en el tiempo, pero el hecho de que se comiencen a cuestionar y a visibilizar alternativas no solo acelera el proceso, sino también permite que personas no conformes al género (Martínez, 2015) encuentren situaciones y modelos identificatorios en los que se puedan sentir reflejadas para aliviar sentimientos de culpa e inadecuación.

\section{Conclusiones}

En lo desarrollado previamente se ha intentado sistematizar y dar cuenta de algunas posibles derivas que se plantean en la actualidad a partir del cuestionamiento y deconstrucción de conceptos tradicionales como género, cuerpo e identidad. También, se han realizado algunos breves recorridos históricos para facilitar que se visibilice la forma en que funciona la construcción social de las significaciones imaginarias sociales y, por consiguiente, de la realidad. Asimismo, se ha señalado cómo la patologización de la diversidad ha tenido un impacto concreto en la vulneración de derechos humanos básicos de las minorías al generar como principal damnificado al colectivo travesti-trans*. Es importante señalar que si bien este escrito invita a repensar la Psicología, sus prácticas y conceptualizaciones, aquí, no se trabaja específicamente sobre esta disciplina. Más bien, se intenta comenzar a deconstruir en el ámbito profesional las nociones generales respecto al género cuerpo e identidad, porque estas son consideradas significaciones de primer orden. Es decir, visibilizar los sentidos que se ponen en juego en:

una significación central que reorganiza, redetermina, reforma una multitud de significaciones sociales ya disponibles, a las que al mismo tiempo altera, condiciona la constitución de otras significaciones y acarrea, lateralmente, efectos análogos prácticamente sobre la totalidad de las significaciones sociales del sistema considerado (Castoriadis, 2013, p. 318).

Al ser significaciones centrales y condicionar todas las prácticas sociales, es importante repensarlas a partir de las modificaciones sociohistóricas que se van presentando para evitar reproducir sentidos que pueden ser antediluvianos e iatrogénicos. Por esta razón, se considera que es fundamental y urgente repensar las conceptualizaciones básicas de la Psicología y de todas las disciplinas en clave de género, así como cuestionarse críticamente el uso de las herramientas teóricas que forman parte de la propia "caja 
de herramientas". Por ejemplo, en el caso de la psicología institucional, podría plantearse que, a partir del recorrido realizado, el concepto de significaciones imaginarias sociales se queda, quizás, demasiado en una dimensión simbólica sin lograr dar cuenta de los aspectos materiales que necesariamente se ponen en jugo al hablar de género, sexo y cuerpo. Es importante complejizar los modos en que se entienden algunos conceptos para poder incluir las múltiples dimensiones que pueden implicar y la necesaria relación que este tipo de elementos establece entre lo social y lo subjetivo, así como entre lo biológico y lo simbólico. En relación con el cuerpo, no se puede pensar solo al organismo o a la imagen corporal que se produce en la psiquis, también es necesario introducir los elementos que articulan la carne con lo social, en tanto que plantea indicios que facilitan o predisponen hacia una lectura social específica de ese cuerpo. Así, las tecnologías estéticas (Castañón-Vázquez, 2018) permiten establecer la forma en que se desea ser percibido y funcionan como intermediarias, porque prestan elementos que facilitan la lectura social que se hace del propio cuerpo y dan cuenta del tipo de relaciones que se desea establecer con el entorno. A partir de la performativización (Butler, 2007) del género las tecnologías estéticas, se constituyen como un habitus (Bourdieu \& Wacquant, 2014) que regula y modeliza los intercambios con el entorno social. Entwistle en El cuerpo y la moda (2002) señala que si bien en las Ciencias Sociales - como la Psicología, los estudios culturales e incluso la misma Sociología - se tiende a separar el vestido del cuerpo. Esto no tiene sentido, porque al hacerlo se fragmenta el objeto de estudio. Es fundamental tratar de dar cuenta de la complejidad del fenómeno que se pretende estudiar y no pueden separarse las dimensiones del cuerpo, la identidad, las tecnologías estéticas y los intercambios sociales. Esta complejización del objeto permite identificar las prácticas y discursos que dan cuenta de las significaciones imaginarias sociales (Castoriadis, 2013) que se ponen en juego en los intercambios sociales y visibilizan el modo en que influyen en la realidad concreta de las personas.

Si bien hasta aquí se intentó sistematizar algo del cuestionamiento propuesto, quedan muchas preguntas que pueden servir de guía para comenzar a construir una psicología crítica, inclusiva, diversa, compleja y situada. Para lograrlo, es necesario incluir a las disidencias desde su diferencia, escucharlas en sus propios términos y tratar de que acompañen a descubrir las posibilidades impensadas que puede deparar el futuro. Algunas de las preguntas que pueden ayudar a pensar algo de esto son la siguientes: ¿Cuáles y cómo son las significaciones imaginarias sociales que se ponen en juego en la construcción del cuerpo de personas trans* y no binarias? ¿Cuáles y cómo son las significaciones imaginarias sociales que se asocian a las tecnologías estéticas utilizadas en la transición? ¿Cómo influyen estas significaciones imaginarias sociales en la construcción de la identidad de las personas trans* y no binarias? ¿En cuáles aspectos estas significaciones imaginarias sociales influyen en la reproducción, la innovación y/o la superación de los patrones dominantes del género?, entre muchas otras posibles.

\section{Referencias}

American Psychiatric Association. (2014). Manual diagnostico y estadístico de los trastornos mentales (5a ed.). Editorial Médica Panamericana.

Arfuch, L. (Comp.) (2002). Identidades, sujetos y subjetividades. Prometeo.

Beauvoir, S. (2017). El segundo sexo. Cátedra. 
CUERPO, GÉNERO E IDENTIDADES

Bleger, J. (1999). Psicohigiene y Psicología Institucional. Paidós.

Bourdieu, P. (2000). La dominación masculina. Anagrama.

Bourdieu, P., \& Wacquant, L. (2014). Una invitación a la sociología reflexiva. Siglo XXI.

Butler, J. (2002). Cuerpos que importan. Sobre los límites materiales y discursivos del "sexo”. Paidós.

Butler, J. (2007). El Género en Disputa. El Feminismo y la Subversión de la Identidad. Paidós.

Carki productions. (2018, 3 de septiembre) ¿Qué es la diversidad sexual? Explicación fácil. [Archivo de

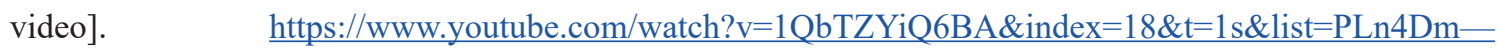
VUFwp5SH13U4iqw2uCBSfrsnjo

Castañón-Vázquez, I. (2018, 7-9 de septiembre). Transgénero y tecnologías estéticas [Ponencia]. XII Congreso Andaluz de Filosofía "Filosofía, Mujeres y Naturaleza", Asociación Andaluza de Filosofía, Sevilla, España.

Castoriadis, C. (2013). La institución imaginaria de la sociedad: Vol. 2. El imaginario social y la institución. Tusquets Editores.

Charmaz, K. (2013). La teoría fundamentada en el siglo XXI: Aplicaciones para promover estudios sobre la justicia social. En N. K. Denzin \& Y. Lincoln (Coord.) Manual de investigación cualitativa. Las estrategias de investigación cualitativa (Vol III., pp. 270-325). Gedisa.

de Lauretis, T. (1989). Technologies of Gender. Essays on Theory, Film and Fiction. Macmillan Press.

Di Segni, S. (2013). Sexualidades: tensiones entre la psiquiatría y los colectivos militantes. Fondo de Cultura Económica.

Entwistle, J. (2002). El cuerpo y la moda. Una visión sociológica. Paidós.

Foucault, M. (1989). Vigilar y Castigar. Siglo XXI Editores.

Foucault, M. (1990). Tecnologías del yo. Paidós.

Foucault, M. (2003). Historia de la sexualidad. La voluntad del saber. Siglo XXI Editores.

Freud, S. (1976). Algunas consideraciones con miras a un estudio comparativo de las parálisis motrices orgánicas e histéricas. En J. L. Etcheverry (Trad.), Obras completas: Sigmund Freud (Vol. I, pp. 191-210). Amorrortu Editores. (Trabajo original publicado en 1893).

Gambarotta, E., \& Mora, A. S. (2018). ¿Cómo se forma un cuerpo? Hacia una problematización sociocultural de la noción de cuerpo desde la tensión naturaleza-cultura. Claroscuro, 17(17), 1-29.

Garosi, E. (2012) "Hacer" lo trans. Estrategias y procesos de transición de género en Turín (Italia). Cuicuilco, 19(54), 139-171.

Hall, S. (2003). ¿Quién necesita 'Identidad'? En S. Hall y P. du Gay (Comp.), Cuestiones de identidad cultural (pp.13-39). Amorrortu editores.

Harawey, D. (1995). Ciencia, cyborgs y mujeres. La reinvención de la naturaleza. Ediciones Cátedra. 


\section{VeLÁZqUeZ}

Kaës, R. (1989). Realidad psíquica y sufrimiento en las instituciones. En R. Kaës (Comp.), La institución y las instituciones. Estudios psicoanalíticos (pp.15-67). Paidós.

Lacan, J. (2003) El estadio del espejo como formador de la función del yo [je] tal como se nos revela en la experiencia psicoanalítica. En Siglo XXI (Eds.), Escritos 1 (pp. 86-93). Siglo XXI.

Laqueur, T. (1994). La construcción del sexo, cuerpo y género desde los griegos hasta Freud. Ediciones Cátedra.

Leibson, L. (2018). La máquina imperfecta. Ensayos del cuerpo en psicoanálisis. Letra viva.

Malfé, R. (1982). Psicología Institucional Psicoanalítica: Superación del Obstáculo Organizacional. Revista Argentina de Psicología, (32), 69-80.

Martínez-Guzmán, A., \& Montenegro, M. (2011). El desafío trans. Consideraciones para un abordaje situado de las identidades de sexo/género. Revista Sociedad \& Equidad, (2), 3-22. https://doi. org/10.5354/0718-9990.2011.14659

Martínez-Guzmán, A. \& Prado, M. A. (2015). Psicología e Identidades Transgénero en el contexto Iberoamericano. Quaderns de Psicologia 17(3), 7-13. https://doi.org/10.5565/rev/qpsicologia.1318

Martínez, A. (2015) Identidad y cuerpo. Auto-percepciones de sujetos no conformes al género [Tesis de doctorado]. Universidad Nacional de La Plata.

Mas-Grau, J. (2017). Del transexualismo a la disforia de género en el DSM. Cambios terminológicos, misma esencia patologizante. Revista Internacional de Sociología 75(2). https://doi.org/10.3989/ris.2017.75.2.15.63

Ministerio de Justicia y Derechos Humanos de la Nación. Secretaría de Derechos Humanos (2012). Ley 26.743 de 2012. Por la cual se establece el derecho a la identidad de género de las personas. https://www.boletinoficial. gob.ar/detalleAviso/primera/70106/20120524

Ministerio Público de la Defensa \& Bachillerato Mocha Celis. (2017). La revolución de las Mariposas. A diez años de La Gesta del Nombre Propio. Ministerio Público de la Defensa.

Organización de Estados Americanos (2018). Comunicado de prensa de la Comisión Interamericana de Derechos Humanos con el motivo del Día Internacional de la Visibilidad Transgénero. http://www.oas.org/es/cidh/ prensa/comunicados/2018/069.asp

Preciado, P. B. (2008). Testo Yonqui. Espasa Calpe.

Red Latinoamericana y del Caribe de Personas Trans. (2020). ;Paren de matarnos! Informe regional 2019-2020. http://redlactrans.org.ar/site/paren-de-matarnos-informe-regional-2019-2020-cedostalc/

Schejter, V. (2018). La construcción de un enfoque epistemológico de la psicología institucional: su implementación en la formación de psicólogos. En V. Schejter, T. Cocha, G. Furlan, y F. Ugo (Comps.), La clínica institucional: construcción compartida de conocimientos (pp. 13-27). Eudeba.

Schiebinger, L. (2004). ¿Tiene sexo la mente? Las mujeres en los orígenes de la ciencia moderna. Ediciones Cátedra.

Ulloa, F. (1995). La Novela clínica Psicoanalítica. Historial de una práctica. Paidós. 
CUERPo, GÉNERO E IDENTIDADES

Wayar, M. (2018). Travesti. Una teoría lo suficientemente buena. Pocas nueces.

Zambrini, L. (2010). Modos de vestir e identidades de género: reflexiones sobre las marcas culturales sobre el cuerpo. Nomadias, (11), 130-149. https://doi.org/10.5354/0719-0905.2010.15158

Recibido: 03 de Marzo de 2020 Revisión recibida: 05 de Octubre de 2021

Aceptado: 09 de Noviembre de 2021

\section{Sobre la autora:}

María Eugenia Velázquez es beneficiaria de una Beca de finalización de doctorado otorgada por el Consejo Nacional de Investigaciones Científicas y Técnicas (CONICET) radicada en el Centro Interdisciplinario de Investigaciones en Género (CInIG) del Instituto de Investigaciones en Humanidades y Ciencias Sociales (IdIHCS) de la Facultad de Humanidades y Ciencias de la Educación (FaHCE) de la Universidad Nacional de La Plata (UNLP). Investigadora becaria en el proyecto "Persona, personae. La metáfora corporal y la representación discursiva de la persona en el seno de las instituciones". Aprobado por resolución N. ${ }^{\circ}$ 059/20 de la Facultad de Ciencias Humanas de la Universidad Nacional de La Pampa (UNLPam). Período de vigencia: 2020-2022. Directora: Lidia Raquel Miranda (Conicet/UNLPam). Dicta clases como docente de la materia Psicología Institucional Cátedra 1 de la Facultad de Psicología de la Universidad de Buenos Aires (UBA). 